\title{
TOPOLOGICALLY TRANSITIVE SKEW-PRODUCTS OF BACKWARD SHIFT OPERATORS AND HYPERCYCLICITY
}

\author{
GEORGE COSTAKIS AND DEMETRIS HADJILOUCAS
}

(Communicated by Joseph A. Ball)

\begin{abstract}
In this article we look at skew-products of multiples of the backward shift and examine conditions under which the skew-product is topologically transitive or hypercyclic in the second coordinate. We also give an application of the theory to iterated function systems of multiples of backward shift operators.
\end{abstract}

\section{INTRODUCTION}

Let $X$ be an infinite dimensional topological vector space either over the field $\mathbb{C}$ or $\mathbb{R}$. A linear continuous operator $T$ acting on $X$ is said to be hypercyclic if there exists a vector $x \in X$ so that its orbit under $T, \operatorname{Orb}(T, x)=\left\{x, T x, T^{2} x, \cdots\right\}$, is dense in $X$. Such a vector $x$ is called hypercyclic for $T$, and $H C(T)$ denotes the set of hypercyclic vectors for $T$. If instead we have a sequence of linear continuous operators $\left\{T_{n}\right\}$ acting on $X$, then the sequence $\left\{T_{n}\right\}$ will be called hypercyclic provided there exists a vector $x \in X$ so that the sequence $\left\{T_{n} x\right\}$ is dense in $X$. Such a vector is called hypercyclic for $\left\{T_{n}\right\}$, and $H C\left(\left\{T_{n}\right\}\right)$ denotes the set of hypercyclic vectors for $\left\{T_{n}\right\}$.

In the literature one may find many examples of hypercyclic operators such as the translation and differentiation operators on the space of entire functions, unilateral and bilateral shifts, and linear differential operators (see the survey articles [10], [11], [17], [6] and [15]). For another approach using ideas from ergodic theory and hypercyclicity, see [2] and [3].

In [16] Rolewicz proved that even though the backward shift operator $B$ : $l^{2}(\mathbb{N}) \rightarrow l^{2}(\mathbb{N})$ on the space of square summable sequences defined by

$$
B\left(x_{1}, x_{2}, x_{3}, \cdots\right)=\left(x_{2}, x_{3}, \cdots\right)
$$

is not hypercyclic, the operator $\lambda B$ is hypercyclic for any $\lambda \in \mathbb{C}$ with $|\lambda|>1$. A related result which came later due to C. Kitai [13] and R. Gethner and J. Shapiro [7] is that, in addition, the set of hypercyclic vectors $H C(\lambda B)$ is $G_{\delta}$ and dense in $l^{2}(\mathbb{N})$.

Throughout this paper we will be using the term backward shift operator for any operator of the form $\lambda B, \lambda \in \mathbb{C}$. In this article, we consider skew-products

Received by the editors August 22, 2006 and, in revised form, November 7, 2006.

2000 Mathematics Subject Classification. Primary 47A16, 28 D99.

Key words and phrases. Hypercyclic operators, skew-product. 
of backward shift operators. Skew-products provide a rich source of dynamical systems whose dynamics vary as the state of the system evolves. One may think of a skew-product as a dynamical system dependent on a parameter that is perturbed as the system evolves in a particular way. The literature on skew-products is very rich; see for instance [1] and [12].

In the present paper we consider a family of backward shift operators generated by an underlying dynamical system with "good" statistical properties. That is, let $(A, \mu)$ be a probability space and $f: A \rightarrow A$ an ergodic map (for a definition see section 2). Also, let $h: A \rightarrow \mathbb{C}$ be an $L^{1}(\mu)$ function. It is then natural to consider, for a complex number $\lambda$, the skew-product $P_{\lambda}: A \times l^{2}(\mathbb{N}) \rightarrow A \times l^{2}(\mathbb{N})$ defined by

$$
P_{\lambda}(\alpha, x)=(f(\alpha), h(\alpha) \lambda B x) .
$$

Note that the product space $A \times l^{2}(\mathbb{N})$ does not have the structure of a linear space, thus $P$ is not a linear operator. Taking the $n^{\text {th }}$ iterate of $P$ gives

$$
P_{\lambda}^{n}(\alpha, x)=\left(f^{n}(\alpha), h\left(f^{n-1}(\alpha)\right) h\left(f^{n-2}(\alpha)\right) \cdots h(f(\alpha)) h(\alpha)(\lambda B)^{n} x\right) .
$$

We write the product in the second coordinate using

$$
h^{n}(\alpha):=h\left(f^{n-1}(\alpha)\right) h\left(f^{n-2}(\alpha)\right) \cdots h(f(\alpha)) h(\alpha) .
$$

Definition 1.1. With maps $f: A \rightarrow A, h: A \rightarrow \mathbb{C}$ as defined above and $\lambda$ a complex number, the skew-product $P_{\lambda}: A \times l^{2}(\mathbb{N}) \rightarrow A \times l^{2}(\mathbb{N})$ defined by $P_{\lambda}(\alpha, x)=$ $(f(\alpha), h(\alpha) \lambda B x)$ is said to be hypercyclic in the second coordinate if the sequence of operators $\left\{h^{n}(\alpha)(\lambda B)^{n}\right\}$ is hypercyclic. Under the additional assumption that $A$ is a topological space, the skew-product $P_{\lambda}$ is said to be topologically transitive if there exists a point $(\alpha, x) \in A \times l^{2}(\mathbb{N})$ so that its orbit under $P_{\lambda}$ is dense in $A \times l^{2}(\mathbb{N})$; i.e., the sequence $\left\{P_{\lambda}^{n}(\alpha, x)\right\}$ is dense.

It is then natural to ask whether and under what conditions a skew-product is hypercyclic in the second coordinate. Furthermore, under the additional assumption that $A$ is a topological space, is it possible to have the stronger property of the existence of a point $(\alpha, x)$ with dense orbit under $P$ in the product space $A \times l^{2}(\mathbb{N})$ ? What structure does the set of points with dense orbit in $A \times l^{2}(\mathbb{N})$ have? We provide answers to the above questions and raise some problems that need further attention.

Let us finally mention that the two main tools in our approach are the ergodic theorem of Birkhoff and Baire's category theorem.

\section{MAIN RESUlT}

In this section we prove our main result. Our purpose is to find sufficient conditions so that skew-products of multiples of the backward shift become topologically transitive. We begin with some definitions.

Definition 2.1. Let $(A, \mathcal{A})$ be a measurable space and $f: A \rightarrow A$ a measurable function. The function $f$ is said to be ergodic with respect to a probability measure $\mu$ on $(A, \mathcal{A})$ if $f$ is measure-preserving with respect to $\mu$ (i.e., $\mu\left(f^{-1}(\Gamma)\right)=\mu(\Gamma)$ for every $\Gamma \in \mathcal{A})$, and $f^{-1}(\Gamma)=\Gamma$ for $\Gamma \in \mathcal{A}$ implies that $\mu(\Gamma) \in\{0,1\}$. The function $f$ is said to be uniquely ergodic if there exists only one probability measure on $(A, \mathcal{A})$ with respect to which $f$ is ergodic.

An important property concerning ergodic maps that will be of use to us is given in the next well-known theorem (see Theorem 5.16 in [18]). 
Theorem 2.2. Let $A$ be a compact metric space, $f: A \rightarrow A$ continuous with $f(A)=A$, and $\mu$ a probability measure on the Borel subsets of $A$ giving nonzero measure to every non-empty open set. If $f$ is an ergodic measure-preserving transformation with respect to $\mu$, then

$$
\mu\left(\left\{\alpha \in A:\left\{f^{n}(\alpha): n=0,1,2, \cdots\right\} \text { is dense }\right\}\right)=1 .
$$

In particular $f$ is topologically transitive.

We are now ready to state and prove our main result.

Theorem 2.3. Let $A$ be a compact metric space, $f: A \rightarrow A$ a continuous map with $f(A)=A, \mu$ an ergodic probability measure on $A$ for $f$ giving non-zero measure to every non-empty open set, and $h: A \rightarrow \mathbb{C}$ a continuous function. For every complex number $\lambda$ consider the skew-product $P_{\lambda}: A \times l^{2}(\mathbb{N}) \rightarrow A \times l^{2}(\mathbb{N})$ defined by

$$
P_{\lambda}(\alpha, x)=(f(\alpha), h(\alpha)(\lambda B) x) .
$$

Suppose

is finite.

$$
\gamma:=\int_{A} \log |h(\alpha)| d \mu
$$

(i) If $|\lambda|>e^{-\gamma}$, then $P_{\lambda}$ is topologically transitive.

(ii) If $|\lambda|<e^{-\gamma}$, then for $\mu$ a.e. $\alpha \in A$, the sequence $\left\{P_{\lambda}^{n}(\alpha, x): n=0,1,2, \cdots\right\}$ is not dense in $A \times l^{2}(\mathbb{N})$ for every $x \in l^{2}(\mathbb{N})$.

(iii) If $f$ is uniquely ergodic and $|\lambda|<e^{-\gamma}$, then $P_{\lambda}$ is not topologically transitive.

Proof. Let

$$
\tilde{A}=\left\{\alpha \in A: \frac{1}{n} \sum_{j=0}^{n-1} \log \left|h\left(f^{j}(\alpha)\right)\right| \rightarrow \int_{A} \log |h(\alpha)| d \mu\right\} .
$$

From the Birkhoff ergodic theorem $\mu(\tilde{A})=1$. Take any complex number $\lambda$ with $|\lambda|>e^{-\gamma}$. Choose a positive number $0<\rho<1$ such that

$$
|\lambda| e^{(1-\rho) \gamma}>1 \text {. }
$$

To show assertion (i) we consider three cases.

Case 1. Suppose $\gamma>0$. For any $\alpha \in \tilde{A}$ there exists $N(\alpha) \in \mathbb{N}$ such that

$$
\left|\frac{1}{n} \sum_{j=0}^{n-1} \log \right| h\left(f^{j}(\alpha)\right)|-\gamma|<\gamma \rho
$$

for all $n \geq N(\alpha)$. Setting

$$
h^{n}(\alpha)=\prod_{j=0}^{n-1} h\left(f^{j}(\alpha)\right)
$$

it follows that

$$
e^{n(1-\rho) \gamma}<\left|h^{n}(\alpha)\right|<e^{n(1+\rho) \gamma}
$$

for all $n \geq N(\alpha)$. Let $X_{1}=\left\{x \in l^{2}(\mathbb{N}): x_{n}=0\right.$ from some $n$ onwards $\}$ which is dense in $l^{2}(\mathbb{N})$. Let $\left\{\left(\alpha_{m}, x_{j}\right)\right\}$ be a countable dense set in $A \times l^{2}(\mathbb{N})$ such that every $x_{j}$ belongs to $X_{1}$. Define the sets

$$
E(m, j, s, n)=\left\{(\alpha, x): d\left(\alpha_{m}, f^{n}(\alpha)\right)<\frac{1}{s},\left\|h^{n}(\alpha)(\lambda B)^{n} x-x_{j}\right\|<\frac{1}{s}\right\}
$$


for $m, j, s, n \in\{1,2, \cdots\}$. We will show that

$$
\bigcup_{n=1}^{\infty} E(m, j, s, n)
$$

is open and dense in $A \times l^{2}(\mathbb{N})$ for every $m, j, s \in\{1,2, \cdots\}$.

Let us first prove that for every $m, j, s, n \in\{1,2, \cdots\}$ the set $E(m, j, s, n)$ is open in $A \times l^{2}(\mathbb{N})$. Fix $m, j, s, n \in\{1,2, \cdots\}$ and let $(\alpha, x) \in E(m, j, s, n)$. It suffices to find an open set $V$ so that $V \subset E(m, j, s, n)$ and $(\alpha, x) \in V$. Since $f$ and $h$ are continuous, there exists $\epsilon>0$ such that

$$
\text { if } d(\alpha, \beta)<\epsilon \text {, then } d\left(f^{n}(\alpha), f^{n}(\beta)\right)<\frac{1}{s}-d\left(\alpha_{m}, f^{n}(\alpha)\right)
$$

and

$$
\text { if } d(\alpha, \beta)<\epsilon \text {, then }\left|h^{n}(\alpha)-h^{n}(\beta)\right|<\frac{\frac{1}{s}-\left\|h^{n}(\alpha)(\lambda B)^{n} x-x_{j}\right\|}{\left\|(\lambda B)^{n} x\right\|+1} .
$$

Define $V:=B_{d}(\alpha, \epsilon) \times B(x, \epsilon)$. Obviously, $(\alpha, x) \in V$ and $V$ is an open set. We show that $V \subset E(m, j, s, n)$. Take $(\beta, y) \in V$. Then using the previous estimates, we get

$$
\begin{aligned}
d\left(\alpha_{m}, f^{n}(\beta)\right) & \leq d\left(\alpha_{m}, f^{n}(\alpha)\right)+d\left(f^{n}(\alpha), f^{n}(\beta)\right) \\
& <d\left(\alpha_{m}, f^{n}(\alpha)\right)+\frac{1}{s}-d\left(\alpha_{m}, f^{n}(\alpha)\right)=\frac{1}{s}
\end{aligned}
$$

and

$$
\begin{aligned}
\left\|h^{n}(\beta)(\lambda B)^{n} x-x_{j}\right\| & \leq\left\|h^{n}(\alpha)(\lambda B)^{n} x-x_{j}\right\|+\left|h^{n}(\alpha)-h^{n}(\beta)\right|\left\|(\lambda B)^{n} x\right\| \\
& <\left\|h^{n}(\alpha)(\lambda B)^{n} x-x_{j}\right\| \\
& +\frac{\frac{1}{s}-\left\|h^{n}(\alpha)(\lambda B)^{n} x-x_{j}\right\|}{\left\|(\lambda B)^{n} x\right\|+1}\left\|(\lambda B)^{n} x\right\| \\
& =\left\|h^{n}(\alpha)(\lambda B)^{n} x-x_{j}\right\|\left(1-\frac{\left\|(\lambda B)^{n} x\right\|}{\left\|(\lambda B)^{n} x\right\|+1}\right) \\
& +\frac{1}{s} \frac{\left\|(\lambda B)^{n} x\right\|}{\left\|(\lambda B)^{n} x\right\|+1} \\
& <\frac{1}{s} \frac{1}{\left\|(\lambda B)^{n} x\right\|+1}+\frac{1}{s} \frac{\left\|(\lambda B)^{n} x\right\|}{\left\|(\lambda B)^{n} x\right\|+1}=\frac{1}{s} .
\end{aligned}
$$

Hence $V \subset E(m, j, s, n)$ and $E(m, j, s, n)$ is open.

To show that each of

$$
\bigcup_{n=1}^{\infty} E(m, j, s, n)
$$

is dense, pick an arbitrary point $(\gamma, y) \in A \times l^{2}(\mathbb{N})$ and $\epsilon>0$. We want to find $(\alpha, x) \in \bigcup_{n=1}^{\infty} E(m, j, s, n)$ such that $d(\alpha, \gamma)<\epsilon$ and $\|x-y\|<\epsilon$. Without loss of generality, take $0<\epsilon<\frac{1}{s}$. Take a point $z=\left(z_{n}\right)_{n \in \mathbb{N}} \in X_{1}$ and a non-negative integer $N_{1}$ such that $\|z-y\|<\epsilon / 2$ and $z_{n}=0$ for all $n \geq N_{1}$. Setting

$$
D:=\left\{\alpha \in A:\left\{f^{n}(\alpha): n=0,1,2, \cdots\right\} \text { is dense in } A\right\},
$$

by Theorem $2.2, \mu(D)=1$. Hence $\mu(D \cap \tilde{A})=1$. Since the set $B_{d}(\gamma, \epsilon)$ is open and it has positive $\mu$ measure, we conclude that

$$
\mu\left(D \cap \tilde{A} \cap B_{d}(\gamma, \epsilon)\right)>0 .
$$


Therefore there exists $\alpha \in D \cap \tilde{A} \cap B_{d}(\gamma, \epsilon)$. Fix such an $\alpha$ and by the previous growth estimate of $\left|h^{n}(\alpha)\right|$ choose $N_{2}(\alpha) \geq N_{1}$ such that

$$
\left|h^{n}(\alpha) \lambda^{n}\right|^{-1}<\frac{\epsilon}{2\left\|x_{j}\right\|} \quad \forall n \geq N_{2}(\alpha) .
$$

Since $\alpha \in D$ we can fix $n \geq N_{2}(\alpha)$ such that

$$
d\left(\alpha_{m}, f^{n}(\alpha)\right)<\epsilon .
$$

Define the forward shift operator $S: l^{2}(\mathbb{N}) \rightarrow l^{2}(\mathbb{N})$ by

$$
S\left(w_{1}, w_{2}, \cdots\right)=\left(0, w_{1}, w_{2}, \cdots\right)
$$

for every $\left(w_{1}, w_{2}, \cdots\right) \in l^{2}(\mathbb{N})$. Setting

$$
x=z+\left(h^{n}(\alpha)\right)^{-1}\left(\frac{1}{\lambda} S\right)^{n} x_{j}
$$

we have

$$
\|x-z\|=\left|h^{n}(\alpha) \lambda^{n}\right|^{-1}\left\|S^{n} x_{j}\right\|=\left|h^{n}(\alpha) \lambda^{n}\right|^{-1}\left\|x_{j}\right\|<\frac{\epsilon}{2},
$$

giving

$$
\|x-y\| \leq\|x-z\|+\|z-y\|<\frac{\epsilon}{2}+\frac{\epsilon}{2}=\epsilon .
$$

Noting that

$$
\left\|h^{n}(\alpha)(\lambda B)^{n} x-x_{j}\right\|=\left\|x_{j}-x_{j}\right\|=0<\frac{1}{s}
$$

it follows that

$$
(\alpha, x) \in E(m, j, s, n)
$$

and has the required property. Baire's category theorem now implies that

$$
\bigcap_{m=1}^{\infty} \bigcap_{j=1}^{\infty} \bigcap_{s=1}^{\infty} \bigcup_{n=1}^{\infty} E(m, j, s, n)
$$

is $G_{\delta}$ and dense in $A \times l^{2}(\mathbb{N})$. It is clear that this is precisely the set of points in $A \times l^{2}(\mathbb{N})$ whose orbit under the skew-product $P_{\lambda}$ is dense.

Case 2. Suppose $\gamma<0$. Then for any $\alpha \in \tilde{A}$ there exists $N(\alpha) \in \mathbb{N}$ such that

$$
\left|\frac{1}{n} \sum_{j=0}^{n-1} \log \right| h\left(f^{j}(\alpha)\right)|-\gamma|<-\gamma \rho
$$

for all $n \geq N(\alpha)$. Setting

$$
h^{n}(\alpha)=\prod_{j=0}^{n-1} h\left(f^{j}(\alpha)\right)
$$

it follows that

$$
e^{n(1+\rho) \gamma}<\left|h^{n}(\alpha)\right|<e^{n(1-\rho) \gamma}
$$

for all $n \geq N(\alpha)$. Then following the same steps as in the previous case, we conclude that $P_{\lambda}$ is topologically transitive. 
Case 3. Suppose $\gamma=0$. Arguing as before, there exists $\delta>0$ such that $|\lambda| e^{-\delta}>1$ and for every $\alpha \in \tilde{A}$ there exists $N(\alpha) \in \mathbb{N}$ such that

$$
|\lambda|^{n} e^{-n \delta}<|\lambda|^{n}\left|h^{n}(\alpha)\right|<|\lambda|^{n} e^{n \delta}
$$

for all $n \geq N(\alpha)$. Following a similar argument as in the previous two cases, we get the desired result. This completes the proof of (i).

To prove assertion (ii), recall our hypothesis $|\lambda|<e^{-\gamma}$. Assume $\gamma>0$. Choose $\epsilon>0$ such that $|\lambda| e^{\epsilon+\gamma}<1$. Then applying Birkhoff's ergodic theorem, we get that for every $\alpha \in \tilde{A}$ there exists $N(\alpha) \in \mathbb{N}$ such that

$$
|\lambda|^{n} e^{n(\gamma-\epsilon)}<|\lambda|^{n}\left|h^{n}(\alpha)\right|<|\lambda|^{n} e^{n(\gamma+\epsilon)}
$$

for all $n \geq N(\alpha)$. Therefore for every $\alpha \in \tilde{A}$ and every $x \in l^{2}(\mathbb{N})$ it follows that $\left|h^{n}(\alpha)\right|\left\|(\lambda B)^{n} x\right\| \rightarrow 0$ as $n$ tends to infinity. The other cases for $\gamma \leq 0$ follow in a similar manner.

To prove (iii), assume $\gamma>0$. Choose $\epsilon>0$ such that $|\lambda| e^{\epsilon+\gamma}<1$. Applying Oxtoby's ergodic theorem, there exists $N \in \mathbb{N}$ such that

$$
\left|\frac{1}{n} \sum_{j=0}^{n-1} \log \right| h\left(f^{j}(\alpha)\right)|-\gamma|<\epsilon
$$

for all $n \geq N$, uniformly for all $\alpha \in A$. Hence

$$
|\lambda|^{n} e^{n(\gamma-\epsilon)}<|\lambda|^{n}\left|h^{n}(\alpha)\right|<|\lambda|^{n} e^{n(\gamma+\epsilon)}
$$

for all $n \geq N$ and for all $\alpha \in A$. Therefore for every $\alpha \in A$ and every $x \in l^{2}(\mathbb{N})$ it follows that $\left|h^{n}(\alpha)\right|\left\|(\lambda B)^{n} x\right\| \rightarrow 0$ as $n$ tends to infinity. Therefore $P_{\lambda}$ is not topologically transitive. The case $\gamma \leq 0$ can be handled in a similar manner. The proof of the theorem is complete.

Remark 2.4. For the case $|\lambda|=e^{-\gamma}$, consider the following example. Let $A=S^{1}=$ $\{z \in \mathbb{C}:|z|=1\}, f: S^{1} \rightarrow S^{1}$ defined by $f\left(e^{2 \pi i \theta}\right)=e^{2 \pi i(\theta+\alpha)}$ for $\alpha \in \mathbb{R} \backslash \mathbb{Q}$, and let $h: S^{1} \rightarrow \mathbb{C}$ be defined by $h\left(e^{2 \pi i \theta}\right)=e^{\gamma}$. Then $f$ is uniquely ergodic, with Haar measure being the unique invariant probability measure, and $h$ is continuous. However, $P_{\lambda}$ is not topologically transitive. On the other hand we have not managed to find an example where $|\lambda|=e^{-\gamma}$ and $P_{\lambda}$ is topologically transitive.

Question 2.5. Under the assumptions of the above theorem and the condition $|\lambda|=e^{-\gamma}$, can $P_{\lambda}$ be topologically transitive?

Remark 2.6. For $h: A \rightarrow \mathbb{C}$ not continuous but $L^{1}(\mu)$, one can have $P_{\lambda}$ hypercyclic in the second coordinate even if $|\lambda|=e^{-\gamma}$. To see this, let $f: S^{1} \rightarrow S^{1}$ be the irrational rotation $f\left(e^{2 \pi i \theta}\right)=e^{2 \pi i(\theta+\alpha)}$ for $\alpha \in \mathbb{R} \backslash \mathbb{Q}$ and choose an arbitrary point $e^{2 \pi i \theta_{0}}$ on $S^{1}$. Define $h\left(e^{2 \pi i \theta_{0}+n \alpha}\right)=\xi$ for $n \in\{0,1,2, \cdots\}, \xi \in \mathbb{C}$ with $|\xi|>1 /|\lambda|$ and $h$ to be $1 /|\lambda|$ everywhere else. Clearly, $\left\{h^{n}\left(\theta_{0}\right)(\lambda B)^{n}\right\}$ is a hypercyclic sequence of operators.

Remark 2.7. Note that the condition $\mu(U)>0$ for any non-empty open set $U$ cannot be omitted. If one takes $f: S^{1} \rightarrow S^{1}$ to be the map $f\left(e^{2 \pi i \theta}\right)=e^{2 \pi i \theta^{2}}$, this gives a uniquely ergodic map which is not topologically transitive on $S^{1}$, thus $P_{\lambda}$ cannot be topologically transitive for any $h: S^{1} \rightarrow \mathbb{C}$. 
Remark 2.8. We would like to point out that the extra assumption of unique ergodicity in statement (iii) of our main result is equivalent to the minimality of $f$ (every orbit is dense) provided $f$ is a homeomorphism (see [18] for details). Therefore, if $f$ is a uniquely ergodic homeomorphism, it is natural to ask whether the following stronger statement holds. For every $\alpha \in A$ there exists an $x \in l^{2}(\mathbb{N})$ such that the sequence $\left\{P_{\lambda}^{n}(\alpha, x)\right\}$ is dense in $A \times l^{2}(\mathbb{N})$.

Example 2.9. Let $A=S^{1}=\{z \in \mathbb{C}:|z|=1\}, f: S^{1} \rightarrow S^{1}$ be an irrational rotation $f\left(e^{2 \pi i \theta_{0}}\right)=e^{2 \pi i\left(\theta_{0}+\theta\right)}$ for some $\theta \in \mathbb{R} \backslash \mathbb{Q}$. Let $h: S^{1} \rightarrow \mathbb{C}$ be defined by

$$
h\left(e^{2 \pi i \theta}\right)=\left(\frac{7}{4}+\sin 2 \pi \theta\right) e^{2 \pi i \theta} .
$$

It is easy to check that $|h| \geq 7 / 4$ for $\theta \in[0,1 / 2]$ and $|h| \geq 3 / 4$ for $\theta \in[1 / 2,1]$, so that

$$
\gamma=\int_{0}^{1} \log |h| d \theta>\frac{1}{2} \log \frac{21}{16}>0,
$$

and so $P_{\lambda}$ with any $\lambda \in \mathbb{C}$ with $|\lambda|=1$ is topologically transitive. However, note that there is a whole interval over which $|h|<1$, and so the multiples of the backward shifts over this interval are contractions. The important thing here is that maps are "expanding on average".

\section{3. $L^{1}$ SKEW-PRODUCT EXTENSIONS AND AN APPLICATION TO ITERATED FUNCTION SYSTEMS}

The following form of the hypercyclicity criterion will prove useful in what follows. There are many versions of this criterion due to several authors (see [8], [5], [4], [14] and [9]), and its final version is due to J. Bès.

Hypercyclicity Criterion. Let $\left\{T_{n}\right\}$ be a sequence of bounded continuous operators acting on a Frechet space $X$. Suppose there exist an increasing sequence $\left\{n_{k}\right\}$ of positive integers, dense subsets $X_{1}, X_{2}$ of $X$ and maps $S_{n_{k}}: X_{2} \rightarrow X_{2}$ such that

(i) $T_{n_{k}} x \rightarrow 0$ as $k \rightarrow+\infty$ for all $x \in X_{1}$,

(ii) $S_{n_{k}} y \rightarrow 0$ as $k \rightarrow+\infty$ for all $y \in X_{2}$,

(iii) $T_{n_{k}} S_{n_{k}} y \rightarrow y$ as $k \rightarrow+\infty$ for all $y \in X_{2}$.

Then the sequence $\left\{T_{n}\right\}$ is hypercyclic.

In the above criterion if $T_{n}=T^{n}$, then we say that $T$ satisfies the hypercyclicity criterion.

Our purpose in this section is to give a sufficient condition for hypercyclicity in the second coordinate under the weaker assumption that $h$ is an $L^{1}$ function. As before, let $(A, \mu)$ be a probability space, $f: A \rightarrow A$ an ergodic map, and $B: l^{2}(\mathbb{N}) \rightarrow l^{2}(\mathbb{N})$ the backward shift on the linear space of square summable sequences. Let $h: A \rightarrow \mathbb{C}$ be an $L^{1}(\mu)$ function.

Theorem 3.1. The skew-product $P: A \times l^{2}(\mathbb{N}) \rightarrow A \times l^{2}(\mathbb{N})$ defined by

$$
P(\alpha, x)=(f(\alpha), h(\alpha) B x)
$$

will be hypercyclic in the second coordinate for $\mu$ a.e. $\alpha \in A$ provided

$$
\int_{A} \log |h(\alpha)| d \mu>0
$$


Proof. An easy application of the Birkhoff ergodic theorem shows that for $\mu$ a.e. $\alpha \in A$, there exists $N(\alpha) \in \mathbb{N}$ such that

$$
e^{n\left(\frac{\gamma}{2}\right)}<\left|h^{n}(\alpha)\right|<e^{n\left(\frac{3 \gamma}{2}\right)}
$$

for all $n \geq N(\alpha)$.

Set $X_{1}=X_{2}=\left\{x \in l^{2}(\mathbb{N}): x_{n}=0\right.$ from some $n$ onwards $\}$ that are dense subsets of $l^{2}(\mathbb{N})$. Furthermore, setting $T_{n}=h^{n}(\alpha) B^{n}, S_{n}=\left(h^{n}(\alpha)\right)^{-1} S^{n}$ (note that $\mu(\{\alpha \in A: h(\alpha)=0\})=0)$ where $S: l^{2}(\mathbb{N}) \rightarrow l^{2}(\mathbb{N})$ is the forward shift defined by $S\left(x_{1}, x_{2}, \cdots\right)=\left(0, x_{1}, x_{2}, \cdots\right)$. It follows that:

(i) $\left\|T_{n} x\right\|=0$ from some $n$ onwards,

(ii) $\left\|S_{n} x\right\|=\left|h^{n}(\alpha)\right|^{-1}\left\|S^{n} x\right\|=\left|h^{n}(\alpha)\right|^{-1}\|x\| \rightarrow 0$ for $\mu$ a.e. $\alpha \in A$ by the previous growth estimate of $h^{n}(\alpha)$,

(iii) $T_{n} \circ S_{n}=I$ where $I$ denotes the identity operator.

Thus it follows from the hypercyclicity criterion that the skew-product $P$ will be hypercyclic in the second coordinate for $\mu$ a.e. $\alpha \in A$.

Remark 3.2. Results such as the above provide a natural generalization to the theorem of Rolewicz. Taking a fixed point $c$ of $f$ with dirac point mass measure $\delta_{c}(C)=1$ if $c \in C$ and $\delta_{c}(C)=0$ otherwise, one obtains the theorem of Rolewicz as a special case where the condition for hypercyclicity

$$
\int_{A} \log |h(\alpha)| d \delta_{c}>0
$$

is simply that $|h(c)|>1$.

Remark 3.3. In the above theorem one may have $\mu\left(h^{-1}\left(B_{1}(0)\right)\right)>0$ where $B_{1}(0)=$ $\{x \in \mathbb{C}:|x| \leq 1\}$ so that a large family (in a measure theoretic sense) of backward shift operators are contraction mappings and hence non-hypercyclic. The crucial thing is that maps are expanding on average.

The same ideas can be used to give an extension to the theorem of Rolewicz in the iterated function scheme. Suppose we have a finite set of backward shift operators $\left\{\lambda_{i} B\right\}_{i=1}^{n}$ with $\lambda_{i} \in \mathbb{C}$ for $i=1, \cdots, n$. Suppose also that each operator is chosen with probability $p_{i}>0, i=1, \cdots, n$, and $\sum_{i=1}^{n} p_{i}=1$. This forms an iterated function system (IFS). We are interested in composing the maps $\left\{\lambda_{i} B\right\}$ with each map $\lambda_{i} B$ chosen randomly with corresponding probability $p_{i}$. We may describe this process using a skew-product as follows. Let $\Omega=\prod_{i=0}^{\infty}\{1, \cdots, n\}$ with product measure $\nu$ induced on cylinder sets from the measure $\tilde{\nu}(i)=p_{i}$ for $i=1, \cdots, n$. Let $T: \Omega \rightarrow \Omega$ be the Bernoulli shift

$$
T\left(w_{0} w_{1} w_{2} \cdots\right)=w_{1} w_{2} w_{3} \cdots
$$

and $P: \Omega \times l^{2}(\mathbb{N}) \rightarrow \Omega \times l^{2}(\mathbb{N})$ be the skew-product

$$
P(w, x)=\left(T(w), \lambda_{w_{0}} B x\right),
$$

where $\lambda_{w_{0}} B$ denotes the backward shift operator corresponding to the digit in the $0^{t h}$ place of the sequence $w$. Throughout we will also be writing $(w)_{0}$ for $w_{0}$, the $0^{t h}$ digit of the sequence $w$. We obtain the following result.

Theorem 3.4. The skew-product $P: \Omega \times l^{2}(\mathbb{N}) \rightarrow \Omega \times l^{2}(\mathbb{N})$, defined by

$$
P(w, x)=\left(T(w), \lambda_{w_{0}} B x\right),
$$


will be hypercyclic in the second coordinate for $\nu$ a.e. $w \in \Omega$ provided

$$
\sum_{i=1}^{n} p_{i} \log \left|\lambda_{i}\right|>0
$$

Proof. The proof is the same as that of Theorem 3.1, and so we just give the first few lines for completeness. As the Bernoulli shift $T: \Omega \rightarrow \Omega$ is ergodic, it follows from the Birkhoff ergodic theorem that

$$
\begin{aligned}
\frac{1}{m} \sum_{j=0}^{m-1} \log \left|\lambda_{\left(T^{j}(w)\right)_{0}}\right| & \rightarrow \int_{\Omega} \log \left|\lambda_{w}\right| d \nu \\
& =\sum_{i=1}^{n} p_{i} \log \left|\lambda_{i}\right|
\end{aligned}
$$

for $\nu$ a.e. $w \in \Omega$. Let $\gamma=\sum_{i=1}^{n} p_{i} \log \left|\lambda_{i}\right|>0$. Then for $\nu$ a.e. $w \in \Omega$ there exists $M(w) \in \mathbb{N}$ such that

$$
\left|\frac{1}{m} \sum_{j=0}^{m-1} \log \right| \lambda_{\left(T^{j}(w)\right)_{0}}|-\gamma|<\frac{\gamma}{2}
$$

for all $m \geq M(w)$. Setting $h^{m}(w)=\prod_{j=0}^{m-1} \lambda_{\left(T^{j}(w)\right)_{0}}$ as before, the proof follows easily in the same way as that of Theorem 3.1.

Example 3.5. Suppose we toss a fair coin repeatedly $(P$ (Heads $)=P$ (Tails $)=1 / 2)$. Each time a Head appears we apply the map $(1 / 2) B$, and each time a Tail appears we apply the map $3 B$. As $p_{1} \log \lambda_{1}+p_{2} \log \lambda_{2}=(1 / 2) \log (3 / 2)>0$, the sequence of operators resulting from such a process will be hypercyclic with probability 1 .

\section{REFERENCES}

[1] L. Arnold, Random Dynamical Systems, Springer Monographs in Mathematics, SpringerVerlag, Berlin, 1998. MR1723992 (2000m:37087)

[2] F. Bayart, S. Grivaux, Hypercyclicity and unimodular point spectrum, J. Funct. Anal. 226 (2005) 281-300. MR2159459 (2006i:47014)

[3] F. Bayart, S. Grivaux, Frequently hypercyclic operators, Trans. Amer. Math. Soc. 358 (2006) 5083-5117. MR2231886 (2007e:47013)

[4] L. Bernal-Gonzalez and K. -G. Grosse-Erdmann, The hypercyclicity criterion for sequences of operators, Studia Math. 157 (2003) 17-32. MR1980114 (2003m:47013)

[5] J. Bès and A. Peris, Hereditarily hypercyclic operators, J. Funct. Anal. 167 (1999) 94-112. MR1710637 (2000f:47012)

[6] J. Bonet, F. Martinez-Gimenez and A. Peris, Linear chaos on Frechet spaces, Dynamical systems and functional equations (Murcia, 2000). Internat. J. Bifur. Chaos Appl. Sci. Engrg. 13 (2003) 1649-1655. MR2015614 (2004i:47016)

[7] R. M. Gethner and J. H. Shapiro, Universal vectors for operators on spaces of holomorphic functions, Proc. Amer. Math. Soc. 100 (1987) 281-288. MR884467 (88g:47060)

[8] G. Godefroy and J. H. Shapiro, Operators with dense invariant cyclic manifolds, J. Funct. Anal. 98 (1991) 229-269. MR1111569 (92d:47029)

[9] S. Grivaux, Hypercyclic operators, mixing operators, and the bounded steps problem, J. Operator Theory 54 (2005) 147-168. MR2168865 (2006k:47021)

[10] K.-G. Grosse-Erdmann, Universal families and hypercyclic operators, Bull. Amer. Math. Soc. (N.S.) 36 (1999) no. 3, 345-381. MR1685272 (2000c:47001)

[11] K.-G. Grosse-Erdmann, Recent developments in hypercyclicity, RACSAM Rev. R. Acad. Cienc. Exactas Fis. Nat. Ser. A Mat. (2003) 273-286. MR2068180 (2005c:47010) 
[12] A. Katok, B. Hasselblatt, Introduction to the modern theory of dynamical systems, Encyclopedia of Mathematics and its Applications, 54, Cambridge University Press, 1995. MR1326374 (96c:58055)

[13] C. Kitai, Invariant closed sets for linear operators, Dissertation, University of Toronto (1982).

[14] F. León-Saavedra, Notes about the hypercyclicity criterion, Math. Slovaca, 53 (2003) 313-319. MR2025025 (2004k:47010)

[15] A. Montes-Rodriguez and H. N. Salas, Supercyclic subspaces, Bull. London Math. Soc. 35 (2003) 721-737. MR2000019 (2004d:47022)

[16] S. Rolewicz, On orbits of elements, Studia Math., 32 (1969) 17-22. MR0241956 (39:3292)

[17] J. H. Shapiro, Notes on the Dynamics of Linear Operators, Unpublished Lecture Notes, (available at www.math.msu.edu/ shapiro).

[18] P. Walters, An introduction to ergodic theory, Graduate Texts in Mathematics, SpringerVerlag, New York-Berlin, 1982. MR648108 (84e:28017)

Department of Mathematics, University of Crete, Knossos Avenue, GR-714 09, HerAklion, Crete, Greece

E-mail address: costakis@math.uoc.gr

The School of Computer Science and Engineering, Cyprus College, 6 Diogenes Street, Engomi, P. O. Box 22006, 1516 Nicosia, Cyprus

E-mail address: dhadjiloucas@cycollege.ac.cy 